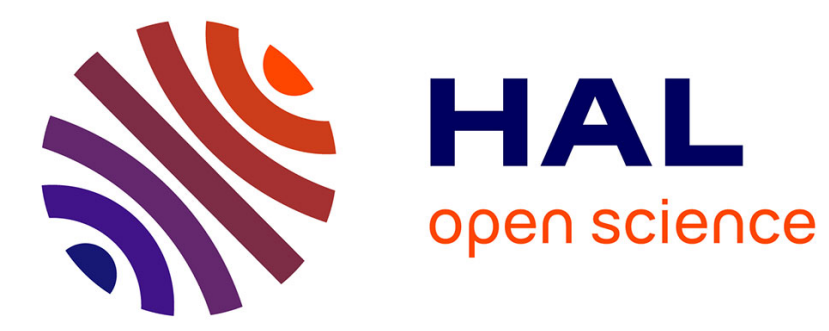

\title{
Experimental analysis of a cantilever beam with a shunted piezoelectric periodic array
}

Benjamin S Beck, Kenneth Cunefare, Massimo Ruzzene, Manuel Collet

\section{To cite this version:}

Benjamin S Beck, Kenneth Cunefare, Massimo Ruzzene, Manuel Collet. Experimental analysis of a cantilever beam with a shunted piezoelectric periodic array. Journal of Intelligent Material Systems and Structures, 2011, 22 (11), pp.1177-1187. 10.1177/1045389X11411119 . hal-02300133

\section{HAL Id: hal-02300133 \\ https://hal.science/hal-02300133}

Submitted on 29 Sep 2019

HAL is a multi-disciplinary open access archive for the deposit and dissemination of scientific research documents, whether they are published or not. The documents may come from teaching and research institutions in France or abroad, or from public or private research centers.
L'archive ouverte pluridisciplinaire HAL, est destinée au dépôt et à la diffusion de documents scientifiques de niveau recherche, publiés ou non, émanant des établissements d'enseignement et de recherche français ou étrangers, des laboratoires publics ou privés. 


\title{
EXPERIMENTAL ANALYSIS OF A CANTILEVER BEAM WITH A SHUNTED PIEZOELECTRIC PERIODIC ARRAY
}

\author{
Benjamin S. Beck \\ Georgia Institute of Technology \\ Woodruff School of Mechanical Engineering \\ Atlanta, GA, USA \\ Kenneth A. Cunefare \\ Georgia Institute of Technology \\ Woodruff School of Mechanical Engineering \\ Atlanta, GA, USA \\ Massimo Ruzzene \\ Georgia Institute of Technology \\ School of Aerospace Engineering \\ Atlanta, GA, USA \\ Manuel Collet \\ FEMTO- ST UMR CNRS \\ Applied Mechanics \\ Atlanta, GA, USA \\ Besançon, France
}




\section{ABSTRACT}

The use of both shunted piezoelectric elements and periodic arrays have been investigated independently as well as used in conjunction to modify the vibration of a system. Piezoelectric patches bonded to a cantilever beam which are shunted with an active circuit, specifically a negative capacitance shunt, can control broadband flexural vibrations of a structure. Also, periodic arrays integrated into a structure allow for modification of propagating waves through the mechanical "stop-bands". The performance of a combined shunted periodic piezoelectric patch array will be analyzed here by investigating the velocity amplitude of the beam upstream and downstream of the array section, and the number of control elements in the array. The negative capacitance shunts caused a global spatial average velocity reduction of $5 \mathrm{~dB}$ at the modal peaks from 500 to $5000 \mathrm{~Hz}$. The reduction is shown to be greater in the downstream section of the beam. Also, by increasing the number of patches in the array the attenuation of the resonances increased nonlinearly. The results show that a negative capacitance periodic control array is an effective global vibration reduction system and has the ability to localize energy near the forcing of a structure. 


\section{INTRODUCTION}

Piezoelectric materials have been studied extensively as a means to reduce vibration and noise. Specifically in this paper, the use of negative capacitance shunts connected to a periodic piezoelectric transducer array applied to a cantilever beam will investigated. Two recent shunt tuning theories have found negative capacitance elements to be necessary for optimal vibration reduction by either reflected wave reduction maximization (Park and Palumbo 2004) or minimization of reactive input power (Cunefare 2006). Theory and experiments have also shown that negative capacitance shunts produce a broadband reduction in vibration amplitude of beam structures (Behrens, Fleming et al. 2001; Park 2005; Park and Baz 2005; Beck, Cunefare et al. 2008).

Forward first suggested the use of an electrical impedance connected across the electrodes of a piezoelectric patch, or shunt, to modify the physical properties of a system to control vibration (Forward 1979). A typical control shunt connected to a piezoelectric patch is shown in Figure 1. Hagood and von Flotow used piezoelectric equations to approximate how a passive (or impedance based active) shunt will modify the material stiffness (Hagood and von Flotow 1991; Park and Palumbo 2004)

$$
E_{p}^{S U}(\omega)=E_{p}^{E} \frac{i \omega C_{p}^{T}+Y^{S U}}{i \omega C_{p}^{T}\left(1-k_{31}^{2}\right)+Y^{S U}}
$$

where $1 / Z_{e}=Y^{S U}$. This equation allowed them to realize that the addition of an inductor would create another degree of freedom. Therefore, the use of a RL shunt demonstrates behavior analogous to a tuned-mass absorber/damper (Hagood and von Flotow 1991; Neubauer, Oleskiewicz et al. 2005). Yet, this type of shunt only has the ability to control vibration at one resonance. Many recent electrical blocking circuit devices have allowed multiple passive resonant shunts connected to one patch to obtain broadband control (Hollkamp 1994; Wu 1998; Fleming and Moheimani 2002; Fleming and Moheimani 2003). Synthetic impedance shunts have been devised that act as active impedance control systems to reduce broadband vibration (Fleming, Behrens et al. 2002; Fleming and Moheimani 2004; Quaegebeur, Micheau et al. 2009).



Figure 1 - Shunted piezoelectric patch with arbitrary impedance 
This paper will focus on negative capacitance shunts which are simple active feedback control circuit. Behrens demonstrated that the shunting of a piezoelectric patch is analogous to feedback control (Behrens, Fleming et al. 2001). Combining this concept with the necessary active operation amplifier within a negative capacitance circuit creates active feedback control which greatly modifies the stiffness and damping of a connected piezoelectric patch to reduce vibration of a system. The value of the negative capacitance is limited by the stability of the circuit (Behrens, Fleming et al. 2001; Park and Baz 2005; Beck, Cunefare et al. 2008; Beck, Cunefare et al. 2009). Therefore, experimentally, the negative capacitance magnitude is set as close to the inherent capacitance of the patch before reaching instability.

Another research area in vibration mitigation is the use of periodic structures. Some of the periodic structures studied for their wave modification include periodic stiffened beams and plates (Mead 1986; Mead and Yaman 1991), SMA inserts (Ruzzene and Baz 2000), and periodic piezoelectric structures (Hofer, Finger et al. 2006; Collet, Cunefare et al. 2009; Spadoni, Ruzzene et al. 2009). These structures have shown creation of "stop-bands" and "pass-bands" which either reduce or allow wave propagation. The elements of these periodic structures can be modified to increase the "stop-band" frequency bandwidth (Baz 2001) therefore enhancing vibration control over a greater frequency range.

The physical effects that a negative capacitance periodic array has on a vibrating beam will be explored by analyzing numerical predictions of wave attenuation through finite elements, an array length study, and the velocity response of different sections of the beam. These investigations will explore how the periodic array enhances the control ability of the negative capacitance shunt. Through the modification of the "stop-bands" of the periodic array, the negative capacitance shunt is expected to have more control over a wider frequency range. The wave attenuation calculations will give perspective to the frequency dependence of the shunt parameters and these parameters will be used as a starting point for the experimental investigations. The array length study will give insight into how much control effort is done with increasing the number of patches. Also, Beck et al. (Beck, Cunefare et al. 2008) remarked that the action of the negative capacitance shunt applied to the boundary of a cantilever beam causes the beam to act as if it is infinite. Therefore, by comparing the velocity response of beam between the forcing and the array, the array area, and between the array and clamp this effect can be further investigated. The results of these studies on a 
periodic beam will give greater insight into the physical effects of the negative capacitance shunted piezoelectric periodic array and how a modal system responds to periodic arrays.

\section{FINITE ELEMENT ANALYSIS}

A numerical study of a coupled mechanical-piezoelectric system is developed to obtain predictions of experimental results. The system of interest is a thin cantilever beam shown in Figure 2. There are 12 equally spaced piezoelectric patch pairs located at the center on the beam, with each patch pair connected to its own individual shunt circuit. These 12 patches constitute the periodic array. The upstream and downstream labels in Figure 2 are such because a point force is located at the tip. The patch array section of beam can be studied by employing a dispersion analysis of single unit cell of the periodic array, Figure 3. The dispersion analysis computes which frequencies are attenuated when a wave is passed through the array. A finite element model for both the full system and the unit cell is developed. The result of interest for the finite element calculations for the beam is the spatial average transverse velocity. The unit cell finite element model obtains the complex wave number that represents the attenuation of propagating waves.

\begin{tabular}{|ccc|}
$\stackrel{2}{4} L_{x}=1.47 \mathrm{~m}$ & \\
\hline Upstream & Array & Downstream \\
\hline$L_{U}=0.421 \mathrm{~m}$ & $L_{D}=0.421 \mathrm{~m}$ \\
\hline
\end{tabular}

Figure 2 - Cantilever beam with a bonded piezoelectric array, centered on beam face

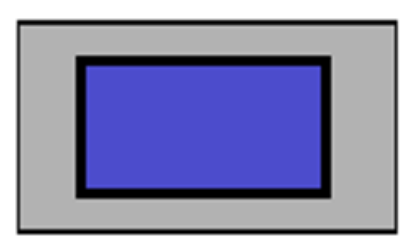

Figure 3 - Single unit cell of periodic array

\section{Energy Functional}

The finite element derivation of the equations of motion for a thin vibrating system is based on the work done by Casadei et al (Casadei, Beck et al. 2009) and Spadoni et al (Spadoni, Ruzzene et al. 2009). The principle of virtual work

$$
\delta L_{t o t}=\delta L_{e x t}-\delta L_{d e f}=0
$$


is first applied to an arbitrary piezoelectric structure shown in Figure 4 . The total work done, $\delta L_{t o t}$, is the difference of both the external and internal forces and the work done by virtual deformation. Rewriting Equation (2) in terms of virtual displacement $\delta u_{i}$ and potential $\delta \phi$ functions and arbitrary space-variables yields

$$
\int_{V_{s}} \delta \varepsilon_{i j} \sigma_{i j, j}-\delta u_{i} \rho \ddot{u}_{i}+\delta \phi D_{i, i} d V=\int_{S_{f}} \delta u_{i} \bar{f} d S-\int_{S_{q}} \delta \phi \bar{q} d S
$$

The forcing terms of Equation (3) include the mechanical dynamic forces, $\bar{f}_{i}$, and the imposed electric charges, $\bar{q}$, which are both defined along the system boundaries, $S_{f}$ and $S_{q}$. The term $u_{i}$ is the mechanical displacement which is defined over the structural domain $V_{S}$. The piezoelectric constitutive equations are needed to obtain the electrical displacement $D_{i}$ and the stress tensor $\sigma_{i j}$ in terms of linear strain $\varepsilon_{k l}$ and electric potential $\phi$. These constructive equations for piezoelectric materials are

$$
\begin{gathered}
\sigma_{i j}=c_{i j k l} \varepsilon_{k l}+e_{k i j} \phi_{k} \\
D_{i}=e_{i k l} \varepsilon_{k l}-\xi_{i k} \phi_{k}
\end{gathered}
$$

where $c_{i j k l}, e_{k i j}$, and $\xi_{i k}$ are material properties. Equations (4) and (5) are substituted into Equation (3) to give the governing equations which are obtained through integration by parts,

$$
\int_{V_{S}} \delta \varepsilon_{i j}\left(c_{i j k l} \varepsilon_{k l}+e_{k i j} \phi_{k}\right) d V+\int_{V_{S}} \delta u_{i} \rho \ddot{u}_{i} d V=\int_{S_{f}} \delta u_{i} \bar{f}_{i} d S
$$

and

$$
\int_{V_{S}} \delta \phi,_{i}\left(e_{i k l} \varepsilon_{k l}-\xi_{i k} \phi,_{k}\right) d V=-\int_{S_{q}} \delta \phi \bar{q} d S
$$

Also, the strain field can be expressed in terms of the displacement variables with the use of the following gradient equation,

$$
\varepsilon_{i j}=\frac{1}{2}\left(u_{i, j}+u_{j, i}\right)
$$

The Kirchhoff kinematic assumptions for thin plate behavior are then applied which state

$$
u_{1}=-z w(x, y)_{x}, u_{2}=-z w(x, y)_{y}, \text { and } u_{3}=w(x, y)
$$

where $w$ is the transverse displacement of the mid-surface of the beam. These conditions result in a plane strain state where the only non-zero components of strain are $\varepsilon_{11}, \varepsilon_{22}$ and $\gamma_{12}$. 


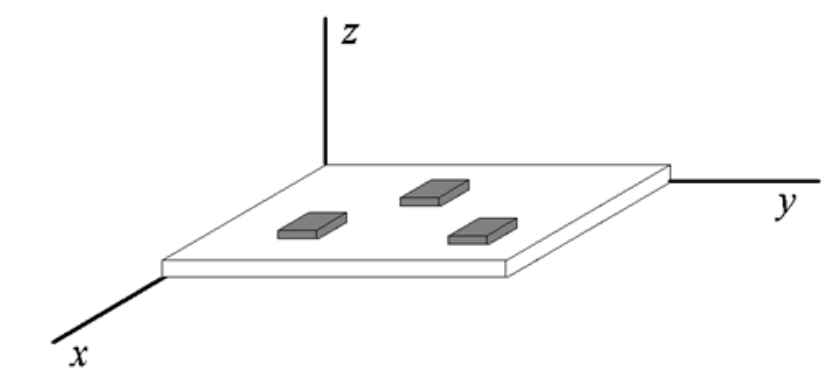

Figure 4 - 2D structure with bonded piezoelectric patches

\section{Finite Element Discretization}

The solution to the governing equations of motion is obtained through a Kirchhoff plate finite element discretization. The use of shape functions and nodal degrees of freedom gives the vertical displacement and surface potential,

$$
w=\left[N_{w}\right]\{d\}
$$

and

$$
\phi=\left[N_{\phi}\right]\{\phi\}
$$

where $N_{w}$ and $N_{\phi}$ are the proper shape functions. The transverse displacement of the mid-surface of the beam at any point within an element is

$$
\begin{aligned}
& w(x, y)=a_{1}+a_{2} x+a_{3} y+a_{4} x y+a_{5} x^{2}+a_{6} y^{2} \\
& +a_{7} x^{2} y+a_{8} x y^{2}+a_{9} x^{3}+a_{10} y^{3}+a_{11} x^{3} y+a_{12} x y^{3}
\end{aligned} .
$$

The $a_{i}$ constants are computed in terms of the nodal variables, $w_{i}, \partial w_{i} / \partial x$, and $\partial w_{i} / \partial y$. Therefore, a pair of matrix equations of motion is obtained by substituting Equations (10) and (11) into Equations (6) and (7) yielding

$$
\left[M_{u u}\right]\{\ddot{d}\}+\left[K_{u u}\right]\{d\}+\left[K_{u \phi}\right]\{\phi\}=\{f\}
$$

and

$$
\left[K_{\phi u}\right]\{d\}+\left[K_{\phi \phi}\right]\{\phi\}=\{q\}
$$

The system mass and stiffness matrices are found using the shape functions and material properties; refer to the work of Cook for exact equations (Cook, Malkus et al. 1989). 
To obtain one equation of motion encompassing both electrical and mechanical degrees of freedom, Equations (13) and (14) are coupled by application of piezoelectric assumptions and a shunting matrix. To start, the electric field at any point on the surface of a piezoelectric patch is considered constant. Also, the electric field potential is assumed to vary linearly through the thickness of a patch. These two assumptions simplify the electrical equation of motion to a scalar equation

$$
\left[K_{u \phi}\right]^{T}\{d\}+K_{\phi \phi} \phi_{p}=q_{e}
$$

The shunting matrix is found by realizing that the electric potential can be expressed in terms of the charge when an arbitrary impedance is connected across the patch electrodes. Assuming a harmonic response, the electric potential is

$$
\phi_{P_{0}}=i \omega Z_{e}(\omega) q_{e_{0}}
$$

where $Z_{e}$ is the shunt impedance. Therefore, combining Equations (13) and (15) produces

$$
\left[\left[K_{u u}\right]-\omega^{2}\left[M_{u u}\right]+\left[S_{Z_{e}}(\omega)\right]\right]\left\{d_{0}\right\}=\left\{f_{0}\right\}
$$

which is the single harmonic motion equation where the shunting matrix is

$$
\left[S_{Z_{e}}(\omega)\right]=i \omega\left[K_{u \phi}\right]\left(i \omega K_{\phi \phi}-\frac{1}{Z_{e}(\omega)}\right)^{-1}\left[K_{\phi u}\right] .
$$

Through inspection of the above equation, the electrical shunt allows for modification of stiffness, mass, and damping of the system depending on the selection of shunt and patch parameters. This formulation will be used to find the velocity frequency response of a complete cantilever beam with a driving point force as well as to analyze a periodic unit cell.

\section{Dispersion Analysis}

As stated earlier, the implementation of a periodic array will modify which wave frequencies will propagate through a structure. To study this physical behavior, the use of a Bloch Theorem analysis is applied to a single repeated cell, Figure 5 (Brioullin 2003). The cell's motion can be found using the above finite element formulas by solving the equation of motion

$$
\left[\left[K_{u u}\right]-\omega^{2}\left[M_{u u}\right]+\left[S_{Z_{e}}(\omega)\right]\right]\{d\}=\left[K_{D}(\omega)\right]\{d\}=\{f\}
$$


where $\left[K_{D}\right]$ is the dynamic stiffness of the element with an applied electrical shunt. Because of the cell periodic, the displacement and forcing at the boundaries of the cell must be continuous from one cell to the next with respect to the wave number. To determine the attenuation of the propagating wave, the wave number must be solved; where the real part of the wave number represents attenuation. To begin, the equations of motion are rewritten in terms of wave number,

$$
\left[K_{D}\left(\mu_{x}, \mu_{y}, \omega\right)\right]^{(r)}\{d\}^{(r)}=0
$$

where $\mu_{x}$ and $\mu_{y}$ are the $x$ and $y$ components of wave number. The solution to this equation is found by holding the frequency and one wave number component constant while solving for the second term, which is equivalent to varying the wave-number vector along the contour of the first irreducible Brioullin zone (Spadoni, Ruzzene et al. 2009). Because of the symmetry of the unit cell, the frequency wave-number period is square as shown in Figure 6. The real positive term that expresses attenuation is called the propagation constant, $\delta$.

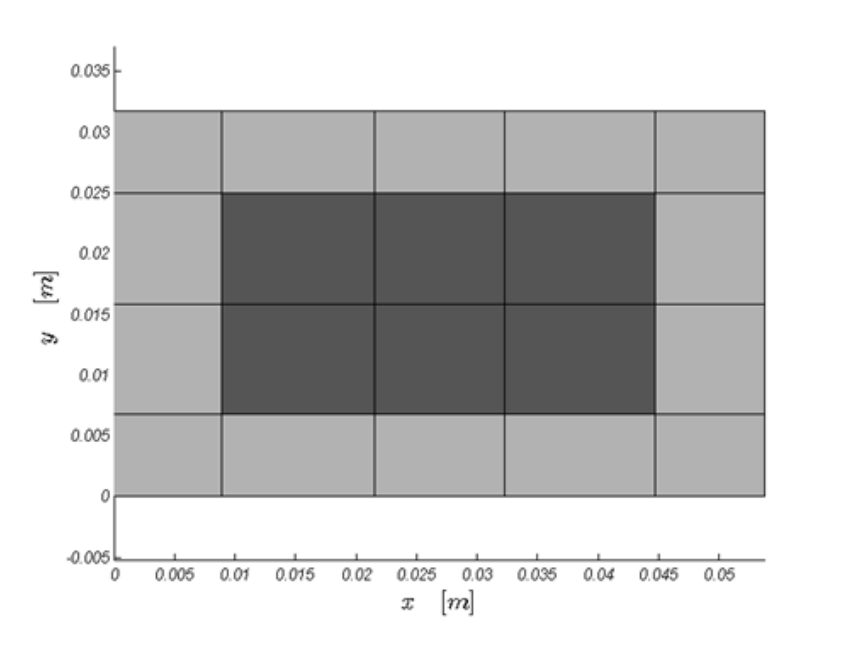

Figure 5 - Schematic of a discretized unit cell 


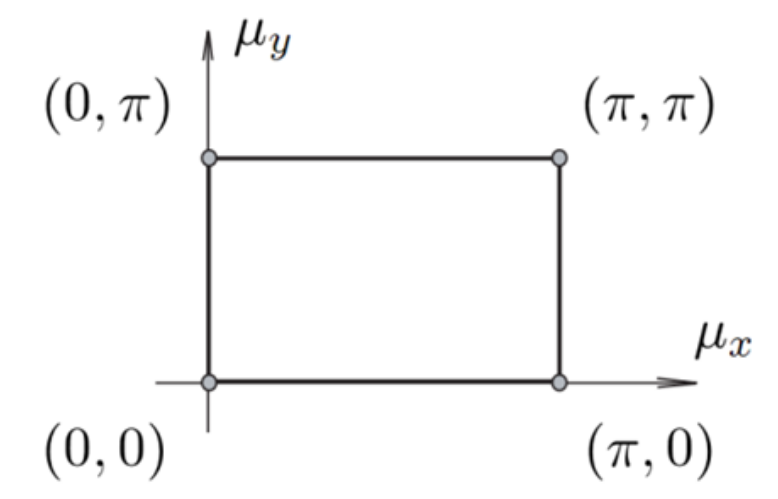

Figure 6 - Sketch of the first irreducable Brioullin zone

\section{EXPERIMENTAL SETUP}

A long cantilever beam with a patch array and necessary data acquisition equipment was assembled to investigate the effect of the negative capacitance shunts. The beam, Figure 2, has been tested in previous work (Beck, Cunefare et al. 2010). A photograph of the beam is shown in Figure 7. The long thin aluminum beam which is clamped on one end has 12 piezoelectric patch pairs bonded symmetrically on both sides, for a total of 24 patches. The construction of the beam consisted of gluing a thin kapton film to both faces of the beam to electrically isolate the bottom surfaces of the piezoelectric patches which are bonded to the kapton. The bottom face of the surface of the piezoelectric was electroded by a thin copper tape that was attached to the piezoelectric by conductive epoxy. The material properties and physical dimensions of the beam and patches are shown in Table 1. Each pair of patches is electrically connected in parallel. The individual pairs are connected to individual shunt circuits which creates 12 single input, single output (SISO) control systems. The beam is also separated into three sections, labeled as: upstream, array, and downstream. These sections will be analyzed separately to investigate force localization.

Figure 7 - Photograph of the cantilever beam with periodic array 
Table 1 - Beam and patch physical parameters

\begin{tabular}{lll}
\hline Beam & Young's modulus & $E_{b}=73 \mathrm{GPa}$ \\
& Density & $\rho_{b}=2700 \mathrm{~kg} / \mathrm{m}^{3}$ \\
& Length & $L=1.47 \mathrm{~m}$ \\
& Width & $w=18.2 \mathrm{~mm}$ \\
& Thickness & $t=3.3 \mathrm{~mm}$ \\
Piezoelectric & Young's modulus, shorted & $E_{p}=63 \mathrm{GPa}$ \\
& Density & $\rho_{p}=7800 \mathrm{~kg} / \mathrm{m}^{3}$ \\
& Coupling coefficient & $k_{31}=0.35$ \\
& Thickness & $t=0.8 \mathrm{~mm}$ \\
& Capacitance & $C_{p}=58.5 \mathrm{nF}$ \\
\hline
\end{tabular}

The negative capacitance shunts used in this work consist of a resistor and a negative capacitance element. The negative capacitance is created using a basic circuit configuration called a negative impedance converter (Horowitz and Hill 1996). The schematic for a negative impedance converter is shown in Figure 8, which consists of three passive circuit elements with impedance $Z_{i}$ and an operational amplifier (op-amp). The equation

$$
Z_{\text {in }}=-\frac{Z_{3} Z_{2}}{Z_{4}}
$$

is used to compute the amount of negative impedance achieved with the implementation of the circuit. To obtain a negative capacitance, a capacitor was placed in the $Z_{2}$ location and a potentiometer was used as resistors in the $Z_{3}$ and $Z_{4}$ locations. The use of a potentiometer allowed for tuning of the circuit with a single reference capacitor, $C_{2}$. Figure 9 is the schematic for the negative capacitance used; the resistor in parallel to the capacitor is necessary for stability of the circuit at DC. At the frequencies of interest, the resistor has negligible impact on the impedance of the shunt. The full shunt diagram is shown in Figure 10. A resistor is added in series with the negative capacitance for positive real impedance. The resistor and negative capacitance are connected in series to the piezoelectric patch. 


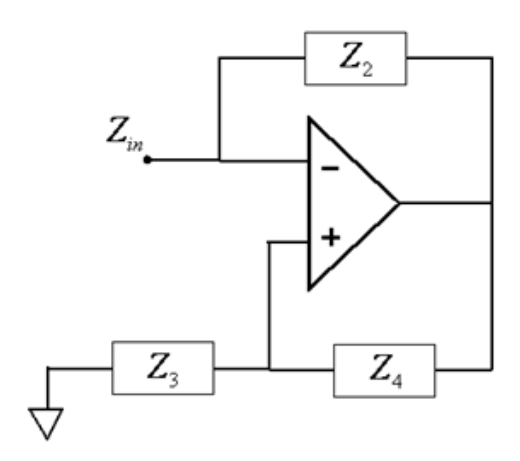

Figure 8 - Negative impedance converter schematic

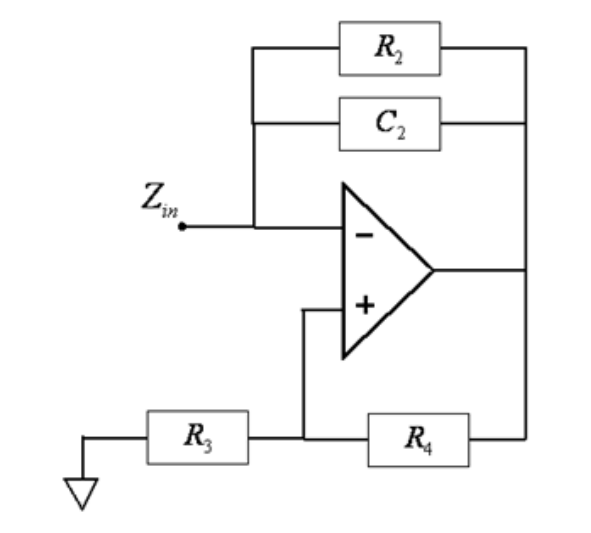

Figure 9 - Negative capacitance schematic

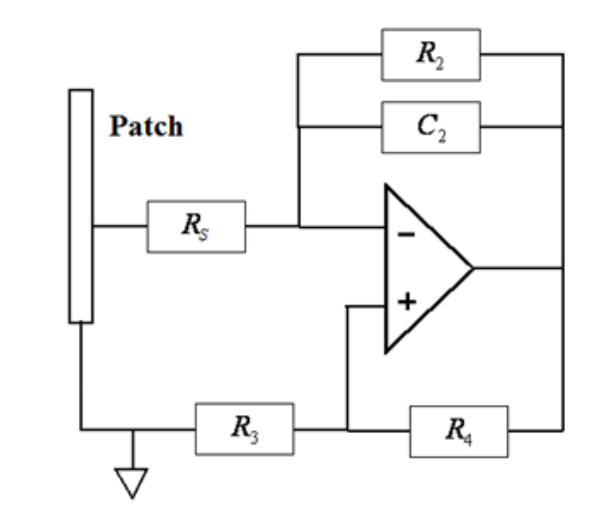

Figure 10 - Full shunt schematic

The response of the cantilever beam is measured by the use of a laser Doppler vibrometer. The vibrometer consists of a computer module, function generator, and scanning laser head. The computer controls the necessary settings for data acquisition and links with the function generator to create a signal output. The output is sent to an 
electromechanical shaker that forces the beam at the tip. The velocity of beam is measured by the scanning laser head at 125 equally spaced locations along the length of the beam. The data from these points are used to compute the spatial average velocity in the regions of interest, either the full length or the three sections of the beam.

\section{RESULTS AND ANALYSIS}

To begin, the results of the dispersion theorem analysis will be presented to express the frequency impact of the control shunt on a unit cell. As stated above, the negative capacitance of the shunt is set to a stable value as close to the magnitude of the patch capacitance. The negative capacitance value is $66.7 \mathrm{nF}$, which will be used for both numerical calculations and experiments. The frequency dependence of the shunt will be explored by changing the resistance in series with the negative capacitance, $R_{2}$ in Figure 10. Figure 11 shows the propagation constant for three values of resistance. For low resistance values, the inherent stop band of the patch geometry at $2600 \mathrm{~Hz}$ is amplified when the circuit is applied. Yet, with higher resistance the increased damping in the shunt minimizes the periodic effect but does increase the control effectiveness at lower frequencies. For the $100 \Omega$ resistance, the propagation constant reaches the same increased stop band level of lower resistance and is greater for both higher and lower frequencies. From this figure, the propagation constant predicts for small resistances the control shunt will have a frequency localized impact. The shunt should have the most effect at lower frequencies with higher resistance.

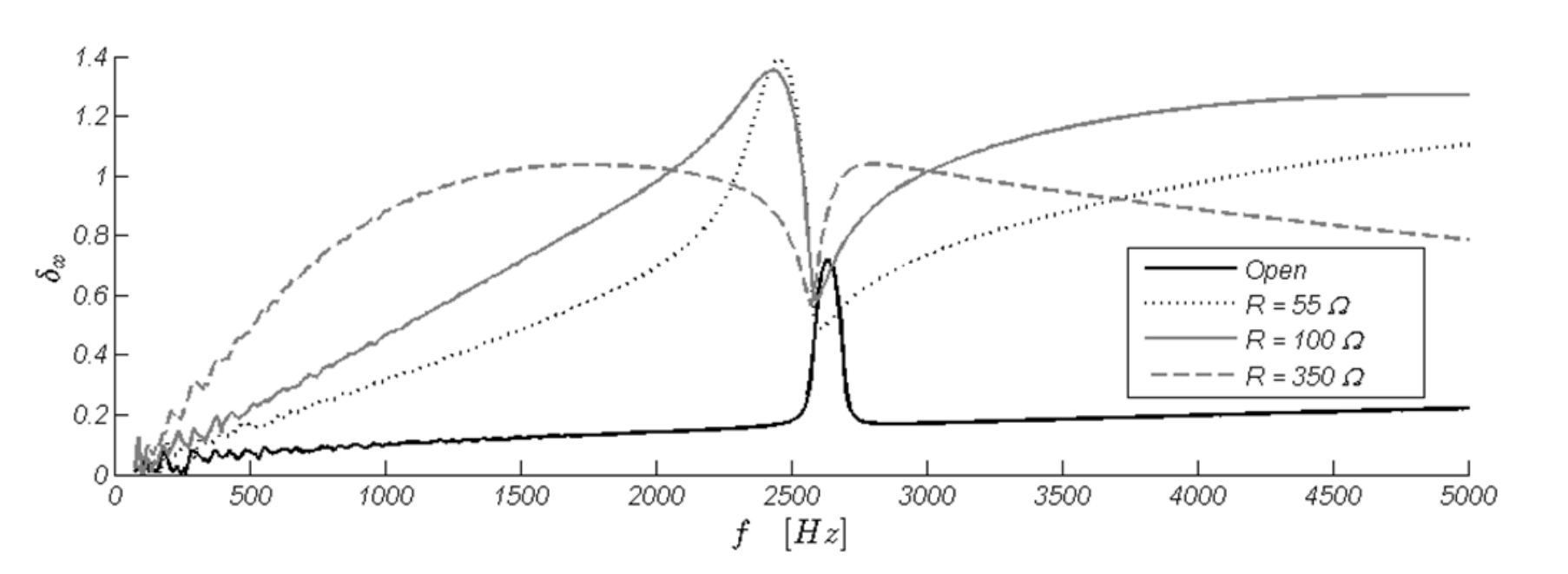

Figure 11 - Propagation constant for negative capacitance shunts 
The spatial average velocity frequency response function (FRF) comparison between a base line (open circuit) case and the control shunt connected expresses an overall reduction in vibration of the beam. The squared spatial average velocity is defined as

$$
\left\langle|u(f)|^{2}\right\rangle=\frac{1}{L_{x}} \int_{0}^{L_{x}} \frac{u^{2}(f, x)}{F(f)} d x
$$

where $f$ is the frequency and $u$ is the velocity at a point $x$ along the length of the beam. Beginning with a finite element simulation, Figure 12, the negative capacitance shunt significantly reduces the velocity of the beam from 600 to $5000 \mathrm{~Hz}$. The maximum numerically predicted reduction is $11 \mathrm{~dB}$ at the $2300 \mathrm{~Hz}$ resonance. The resonance at $2500 \mathrm{~Hz}$ cannot be controlled by the shunts, but both resonances above and below this exhibit maximum attenuation. This behavior agrees with the dispersion analysis results where the effectiveness dips at $2500 \mathrm{~Hz}$ but has a maximum at the frequencies immediately lower. As was also predicted in Figure 11, the frequency dependant behavior is obtained from the finite element model. The $100 \Omega$ resistor creates the largest attenuation at frequencies above the uncontrolled mode. In addition, the high resistance configuration produces the largest control at low frequencies. The numerical spatial average velocity response is verified experimentally as shown in Figure 13. Both the propagation constant predictions and the numerical results are confirmed by the experimental velocity response of the beam. Figure 13 shows the spatial average velocity of the beam for the same three values of resistance. The resonance at $2600 \mathrm{~Hz}$ is also not controlled. Yet, the maximum suppression is found to be $12 \mathrm{~dB}$ at $2100 \mathrm{~Hz}$ for the $100 \Omega$ resistor. The frequency nature of the three shunt configurations can be seen as well, with the $55 \Omega$ resistor having a strong localized effect around $2400 \mathrm{~Hz}$. A minimum of $5 \mathrm{~dB}$ velocity reduction is obtained at all modal peaks from 600 to $5000 \mathrm{~Hz}$ with the $100 \Omega$ resistor. It should also be noted that these attenuation values represent a global measure for the reduction in vibration amplitude because $\langle|u|\rangle$ is a spatial average over the entire length. 


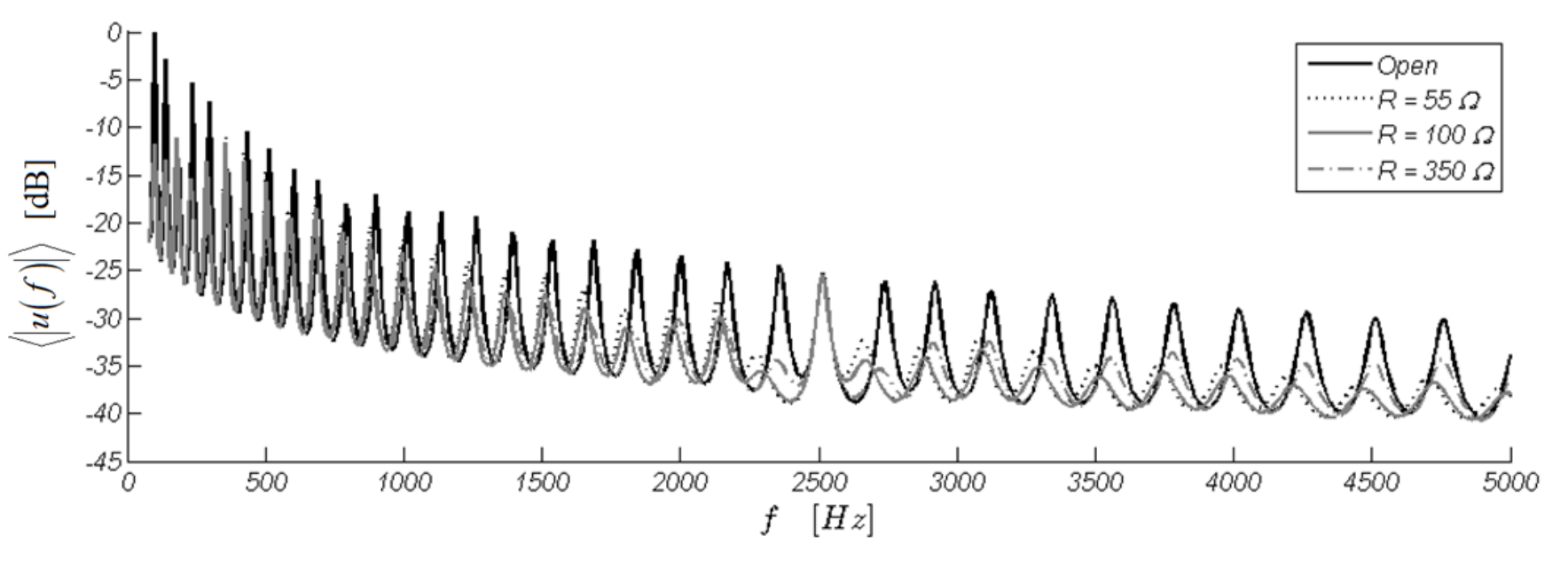

Figure 12 - Finite element numerical spatial average velocity FRF

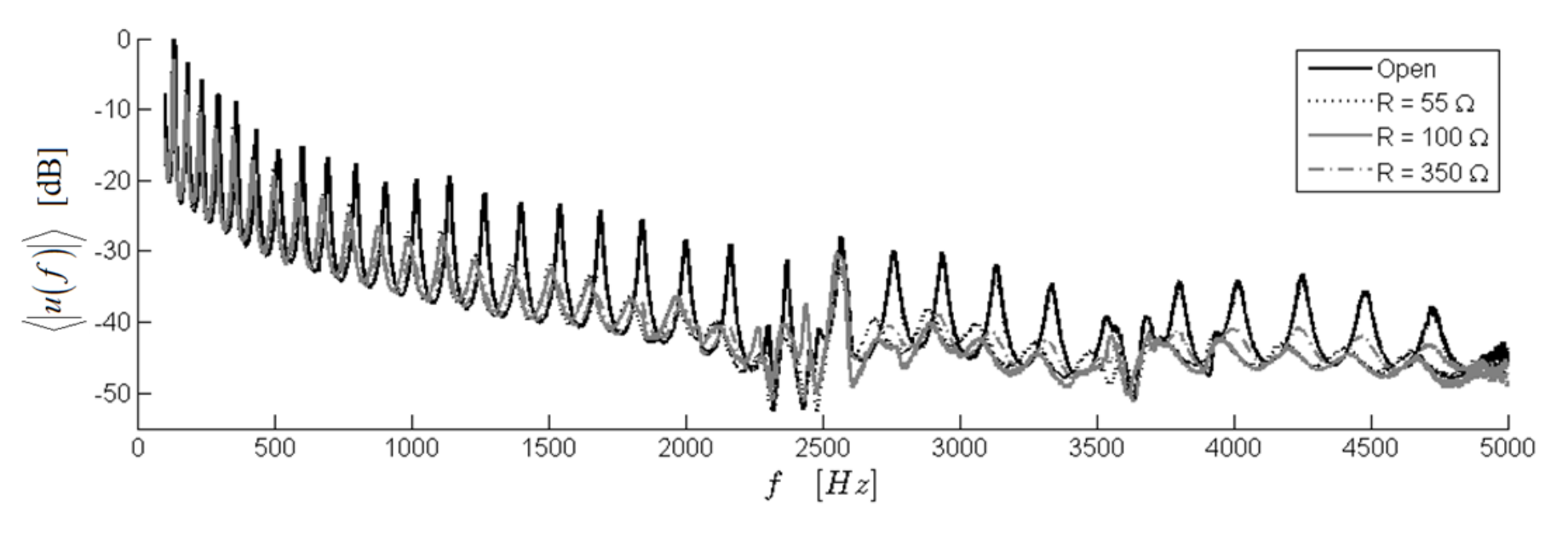

Figure 13 - Experimental spatial average velocity FRF

The three sections of the beam behave differently when the control shunt is applied. Figure 14 and Figure 15 show the spatial average velocity response of the upstream and downstream sections of the beam. The negative capacitance control causes the velocity minima to increase in the upstream section of the beam as seen in Figure 14. In contrast, Figure 15 shows the average velocity decreases at the anti-resonances of the downstream section. These changes are caused by the vibration localization of the shunt array. This can be quantified by summing the spatial average kinetic energy of each section over all frequencies. The frequency integrated kinetic energy difference between the open and control cases is defined as

$$
\Delta T=10 \log _{10}\left[\int_{f}^{f_{\max }} \frac{T_{c}}{T_{u c}} d f\right]
$$


where $T_{c}$ is the controlled kinetic energy and $T_{u c}$ is the uncontrolled kinetic energy. The kinetic energy change between the open circuit and control cases for the upstream and downstream sections of the beam are shown in Table 2. This table displays the difference between uncontrolled and controlled kinetic energy for three lower integration limits. As the integration is confined to higher frequencies, the greater the difference between the controlled and uncontrolled cases. This shows that the shunt reduces the energy of the vibration as the waves pass through the array. Therefore for higher frequencies, the circuit has a greater vibration localization effect. This trend is expressed in Figure 16, also. The two curves show the kinetic energy difference for the upstream and downstream sections of the beam. The separation between the two curves increases as the frequency of the lower integration limit increases. In summary, the spatial average velocity of the whole beam decreased when the control is added, but the array has a greater effect in reducing the energy in the area downstream of the array. The effect of the array can also be considered an anechoic boundary, because the vibration energy is dissipated and not reflected back upstream.

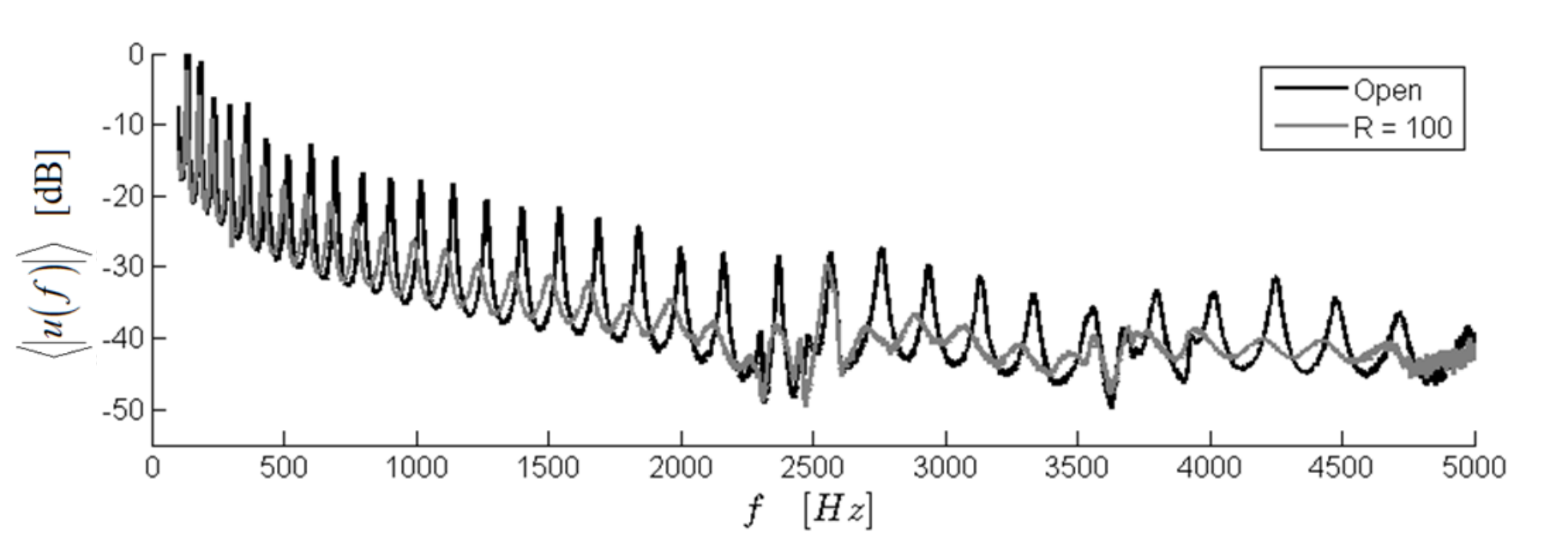

Figure 14 - Spatial average velocity FRF for left section 




Figure 15 - Spatial average velocity FRF for right section

Table 2 - Frequency integrated total kinetic energy difference

\begin{tabular}{c|cc}
\hline Range & Upstream & Downstrem \\
\hline $0.1-5 \mathrm{kHz}$ & $-4.2 \mathrm{~dB}$ & $-5.7 \mathrm{~dB}$ \\
\hline $0.5-5 \mathrm{kHz}$ & $-6.6 \mathrm{~dB}$ & $-10.4 \mathrm{~dB}$ \\
\hline $1.5-5 \mathrm{kHz}$ & $-6.2 \mathrm{~dB}$ & $-13.7 \mathrm{~dB}$
\end{tabular}

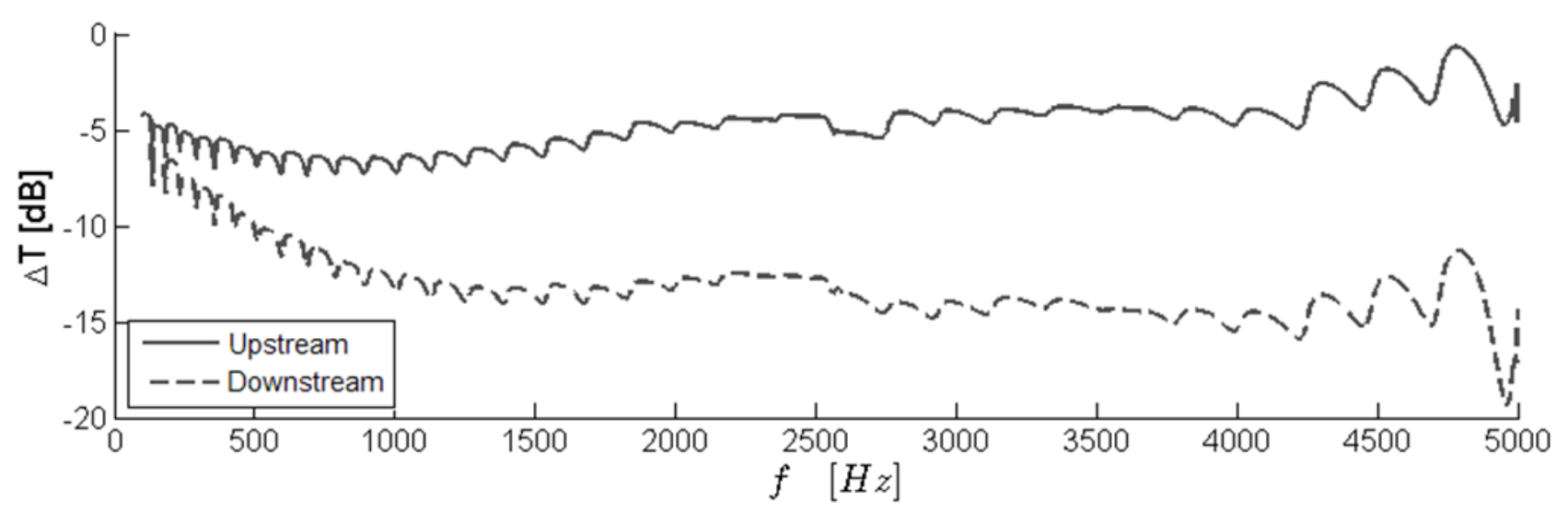

Figure 16 - Frequency integrated kinetic energy difference

The number of patches with the control connected impacts the reduction in velocity of the resonant peaks. Figure 17 shows the numerical prediction of attenuation for the $15^{\text {th }}$ and $16^{\text {th }}$ resonant peaks of the beam relative to the open circuit case as more patches are added to the array length. The $15^{\text {th }}$ and $16^{\text {th }}$ resonances are located at 1400 and $1550 \mathrm{~Hz}$. A pair of sequential resonances was needed to analyze the behavior of the control when increasing the array length, and these two were chosen arbitrarily with average modal attenuation. The overall trend is an increasing reduction with increasing array length, yet the addition of certain patches has more control than others. Also, the modes are affected differently when more patches are applied. The attenuation increases by increasing the 
number of patches in the array; but not smoothly increasing, rather, exhibiting ramps and plateaus. The behavior is attributed to the modal response of the beam, which affects the locations where a patch can exert control. The ramps are present when the addition of a single patch causes significantly more control to the overall vibration. In contrast, a plateau occurs when adding another patch to the array length does not cause any increase in attenuation. The reduction in modal amplitude therefore depends on the location of the additional patch in the array with respect to the mode shape. Therefore, the propagation constant cannot be considered an exact prediction between reduction in vibration of a modal system at every frequency.

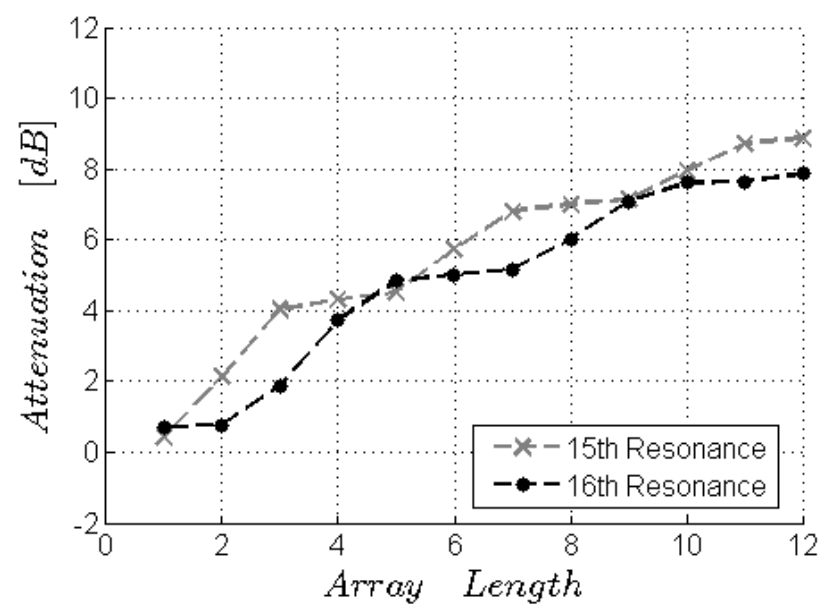

Figure 17 - Numerical vibration reduction versus array length, \# of patches

The experimental data shows a similar ramps and plateaus in the increasing attenuation with increased array length, Figure 18. The corresponding resonances show after 8 patches there is only incremental increases in attenuation. Therefore, more attenuation may not be possible with a longer array. 


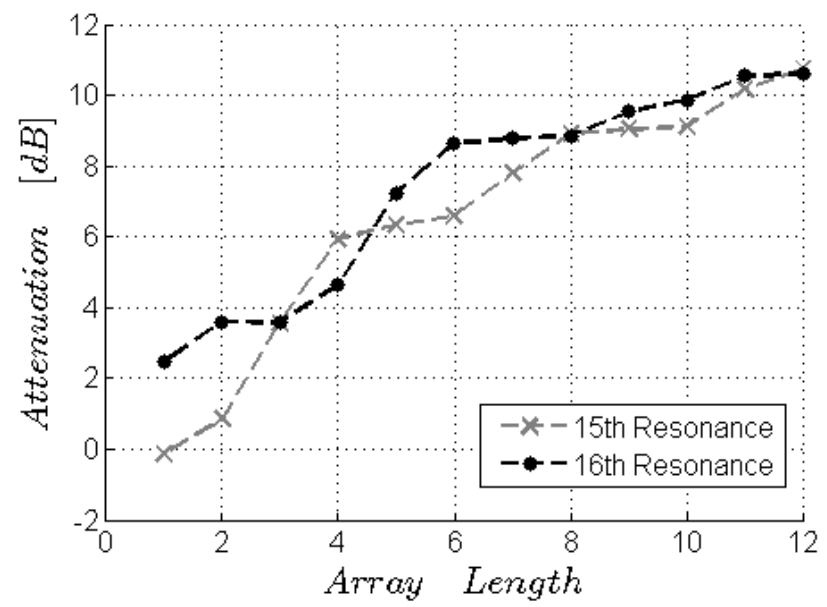

Figure 18 - Experimental velocity reduction versus array length, \# of patches

\section{CONCLUSION}

The effect of the negative capacitance shunted piezoelectric patch on a cantilever beam is a reduction in overall vibratory response. A dispersion analysis approximates the frequency dependence effect of the shunt, but the propagation constant does not give a precise measure for each resonance of a modal system. Yet, increasing array length causes an increase in vibration control, with a maximum of $12 \mathrm{~dB}$. The control array also results in the beam to begin to act as if it there is an anechoic termination where more of the vibration energy is localized between the forcing location and the patch array.

\section{ACKNOWLEDGMENTS}

This work is supported by a collaborative research agreement [NNX07AD20A] between NASA Langley Research Center and the Georgia Institute of Technology.

\section{REFERENCES}

Baz, A., 2001. "Active Control of Periodic Structures," Transactions of the ASME. Journal of Vibration and Acoustics 123(10): 472-479.

Beck, B., Cunefare, K. A., et al., 2008. Experimental Assessment of Negative Impedance Shunts for Vibration Suppression on a Beam. In: Proceedings of SPIE - Smart Structures and Materials March 9-13, San Diego, CA. Vol. 6928 
Beck, B., Cunefare, K. A., et al., 2010. Periodic Piezoelectric Sensor-Actuator Array for Vibration Suppression on a Beam. In: Proceedings of SPIE - Smart Structures and Materials March 9-12, San Diego, CA. Vol. 7643

Beck, B., Cunefare, K. A., et al., 2008. Broadband Vibration Suppression Assessment of Negative Impedance Shunts. In: Proceedings of SMASIS08, October 28-30, Ellicott City, Maryland USA. Vol.

Beck, B., Cunefare, K. A., et al., 2009. Experimental implementation of negative impedance shunts on periodic structures. In: Proceedings of SMASIS09, September 20-24, Oxnard, CA USA. Vol.

Behrens, S., Fleming, A. J., et al., 2001. New method for multiple-mode shunt damping of structural vibration using a single piezoelectric transducer. In: Proceedings of SPIE - Smart Structures and Materials 2001: Damping and Isolation, 5-7 March 2001, /, Newport Beach, CA, USA. Vol. 4331

Brioullin, L., 2003. Wave propagation in periodic structures, Dover.

Casadei, F., Beck, B., et al., 2009. Vibration Control of Plates Featuring Periodic Arrays of Hybrid Shunted Piezoelectric Patches. In: Proceedings of SPIE - Smart Structures and Materials, San Diego, CA, USA. Vol. 7288

Collet, M., Cunefare, K. A., et al., 2009. "Wave Motion Optimization in Periodically Distributed Shunted Piezoelectric Beam Structures," Journal of Intelligent Material Systems and Structures 20(7): 787 - 808.

Cook, R. D., Malkus, D. S., et al., 1989. Concepts and Applications of Finite Element Analysis. New York, NY, Wiley.

Cunefare, K. A., 2006. Negative capacitance shunts for vibration suppression: wave based tuning and reactive input power. In: Proceedings of Active 2006, September, Adelaide, Australia. Vol.

Fleming, A. J., Behrens, S., et al., 2002. "Optimization and implementation of multimode piezoelectric shunt damping systems," IEEE/ASME Transactions on Mechatronics 7(1): 87-94.

Fleming, A. J. and Moheimani, S. O. R., 2002. Adaptive piezoelectric shunt damping. In: Proceedings of SPIE Smart Structures and Materials 2002: Modeling, Signal Processing, and Control, 18-21 March 2002, /, San Diego, CA, USA. Vol. 4693

Fleming, A. J. and Moheimani, S. O. R., 2003. "Adaptive piezoelectric shunt damping," Smart Materials and Structures 12(1): 36-48. 
Fleming, A. J. and Moheimani, S. O. R., 2004. Optimal Impedance Design for Piezoelectric Vibration Control. In: Proceedings of 43rd IEEE Conference on Decision and Control, December 14-17, Atlantis, Paradise Island, Bahamas. Vol.

Forward, R. L., 1979. "Electronic damping of vibrations in optical structures," Applied Optics 18: 690-697.

Hagood, N. W. and von Flotow, A., 1991. "Damping of structural vibrations with piezoelectric materials and passive electrical networks," Journal of Sound and Vibration 146(2): 243-268.

Hofer, M., Finger, N., et al., 2006. "Finite-Element Simulation of Wave Propagation in Periodic Piezoelectric SAW Structures," IEEE Transactions on Ultrasonics, Ferroelectrics, and Frequency Control 53(6): 1192-1201.

Hollkamp, J. J., 1994. "Multimodal passive vibration suppression with piezoelectric materials and resonant shunts," Journal of Intelligent Material Systems and Structures 5(1): 49-57.

Horowitz, P. and Hill, W., 1996. The Art of Electronics. New York, NY, Cambridge University Press.

Mead, D. J., 1986. "A New Method of Analyzing Wave Propagation in Periodic Structures; Applications to Periodic Timoshenko Beams and Stiffened Plates," Journal of Sound and Vibration 114: 9-27.

Mead, D. J. and Yaman, Y., 1991. "The response of infinite periodic beams to point harmonic forces: A flexural wave analysis," Journal of Sound and Vibration 144(3).

Neubauer, M., Oleskiewicz, R., et al., 2005. "Comparison of Damping Performance of Tuned Mass Dampers and Shunted Piezo Elements," PAMM 5(1): 117-118.

Park, C. H., 2005. Multi-mode vibration damping device and method using negative capacitance shunt circuits. United States.

Park, C. H. and Baz, A., 2005. "Vibration control of beams with negative capacitive shunting of interdigital electrode piezoceramics," Journal of Vibration and Control 11: 331-346.

Park, J. and Palumbo, D. L., 2004. A New Approach to Identify Optimal Properties of Shunting Elements for Maximum Damping of Structural Vibration Using Piezoelectric Patches. In: Proceedings of Active 2004, Williamsburg, VA, CD Proceedings published by the Institute of Noise Control Engineers. Vol.

Quaegebeur, N., Micheau, P., et al., 2009. "Decentralized harmonic control of sound radiation and transmission by a plate using a virtual impedance approach," Journal of the Acoustical Society of America 125(5): 2978-2986.

Ruzzene, M. and Baz, A., 2000. "Control of Wave Propagation in Periodic Composite Rods Using Shape Memory Alloys," Transactions of the ASME. Journal of Vibration and Acoustics 122(4): 151-159. 
Spadoni, A., Ruzzene, M., et al., 2009. "Vibration and Wave Propagation Control of Plates with Periodic Arrays of Shunted Piezoelectric Patches," Journal of Intelligent Material Systems and Structures 20(8): 979-990.

Wu, S.-Y., 1998. "Method for multiple mode piezoelectric shunting with single PZT transducer for vibration control," Journal of Intelligent Material Systems and Structures 9(12): 991-998. 